\title{
Lithium-mediated protection against ethanol neurotoxicity
}

\author{
Jia Luo* \\ Department of Internal Medicine, University of Kentucky College of Medicine, Lexington, KY, USA
}

\section{Edited by:}

Xiao-Ming Ou, University of Mississippi

Medical Center, USA

Reviewed by:

Jordi Calderó, Universitat de Lleida and

Institut de Recerca Biomedica de

Lleida, Spain:

Xiao-Ming Ou, University of Mississippi

Medical Center, USA

\section{*Correspondence}

Jia Luo, Department of Internal Medicine, University of Kentucky College of Medicine, 130 Bosomworth Health Sciences Research Building, 1095 Veterans Drive, Lexington, $K Y$ 40536, USA.

e-mail: jialuo888@uky.edu
Lithium has long been used as a mood stabilizer in the treatment of manic-depressive (bipolar) disorder. Recent studies suggest that lithium has neuroprotective properties and may be useful in the treatment of acute brain injuries such as ischemia and chronic neurodegenerative diseases such as Alzheimer's disease, Parkinson's disease, Huntington's disease and amyotrophic lateral sclerosis. One of the most important neuroprotective properties of lithium is its anti-apoptotic action. Ethanol is a neuroteratogen and fetal alcohol spectrum disorders (FASD) are caused by maternal ethanol exposure during pregnancy. FASD is the leading cause of mental retardation. Ethanol exposure causes neuroapoptosis in the developing brain. Ethanol-induced loss of neurons in the central nervous system underlies many of the behavioral deficits observed in FASD. Excessive alcohol consumption is also associated with Wernicke-Korsakoff syndrome and neurodegeneration in the adult brain. Recent in vivo and in vitro studies indicate that lithium is able to ameliorate ethanol-induced neuroapoptosis. Lithium is an inhibitor of glycogen synthase kinase 3 (GSK3) which has recently been identified as a mediator of ethanol neurotoxicity. Lithium's neuroprotection may be mediated by its inhibition of GSK3. In addition, lithium also affects many other signaling proteins and pathways that regulate neuronal survival and differentiation. This review discusses the recent evidence of lithium-mediated protection against ethanol neurotoxicity and potential underlying mechanisms.

Keywords: alcohol, apoptosis, development, fetal alcohol syndrome, neurodegeneration, neuroprotection

\section{INTRODUCTION}

Lithium is a classic mood stabilizer and it was the first drug approved by the Food and Drug Administration (FDA) in 1974 for maintenance treatment of bipolar disorder (Pies, 2002). There has been a resurgence of interest in lithium due to studies that suggest lithium has neurotrophic and neuroprotective properties. Recent advances in cellular and molecular biology have shown that it may be useful in the treatment of acute brain injuries such as ischemia and chronic neurodegenerative diseases such as Alzheimer's disease, Parkinson's disease (PD), Huntington's disease (HD) and amyotrophic lateral sclerosis (ALS) (Marmol, 2008). Lithium has demonstrated diverse molecular effects reversing well-described pathophysiological changes such as increased oxidative stress, apoptosis, inflammation, environmental stress, glial dysfunction, neurotrophic factor deficiency, excitotoxicity as well as mitochondrial and endoplasmic reticulum disruption (Machado-Vieira et al., 2009). One of the most important neuroprotective properties of lithium is its antiapoptotic action (Bielecka and Obuchowicz, 2008).

Fetal Alcohol Spectrum Disorders (FASD) are caused by maternal alcohol consumption during pregnancy and characterized by a spectrum of structural anomalies and neurocognitive and behavioral disabilities (Riley and McGee, 2005). FASD currently represent the leading cause of mental retardation (Stratton et al., 1996; May and Gossage, 2001; Nash et al., 2008). Prominent central nervous system (CNS) abnormalities in FASD include: microencephaly, abnormal cortical thickness, reduced cerebral white matter volume, ventriculomegaly and cerebellar hypoplasia (Clarren et al., 1978; Danis et al., 1981; Swayze et al., 1997; Archibald et al., 2001; Bookstein et al., 2006; Sowell et al., 2008). These alcohol-induced structural alterations in the brain underlie many of the behavioral deficits observed in FASD. Experimental models have been successfully used to investigate teratogenic effects of ethanol, and CNS abnormalities in FASD are replicated in experimental animal models. Neuronal loss is the most severe consequence of developmental exposure to ethanol. Chronic binge alcohol exposure also induces neurodegeneration in the adult brain and is associated with neurocognitive deficits and Wernicke-Korsakoff syndrome (Obernier et al., 2002; Pfefferbaum et al., 2007; Harper, 2009). It is therefore important to identify potential neuroprotective agents that can ameliorate ethanol-induced CNS damage. This review discusses the evidence of lithium-mediated protection against ethanol neurotoxicity and potential underlying mechanisms.

\section{EVIDENCE OF LITHIUM-MEDIATED PROTECTION AGAINST ETHANOL NEUROTOXICITY}

The development of the rodent brain during early postnatal days (the brain growth spurt) corresponds to that of the human third trimester and ethanol exposure during this period causes neurodegeneration (Olney et al.,2000). Injection of ethanol to C57BL/6 mice on postnatal day 7 (PD7) is a widely used animal model; acute ethanol exposure causes widespread apoptotic neurodegeneration in the brain of PD7 mice (Olney et al., 2000; Young and Olney, 2006). Using this model system, investigators independently demonstrated that lithium was able to protect ethanol-induced neuroapoptosis (Zhong et al., 2006; Chakraborty et al., 2008; Young et al., 2008; Liu et al., 2009). In our study (Liu et al., 2009), PD7 C57BL/6 mice were injected subcutaneously with saline or ethanol ( $20 \%$ solution in saline, $2.5 \mathrm{~g} / \mathrm{kg})$ at $0 \mathrm{~h}$ and $2 \mathrm{~h}$, and lithium was administrated intraperitoneally $30 \mathrm{~min}$ prior to ethanol exposure. Lithium effectively blocked ethanol-induced activation of caspase- 3 and Bax in the developing cerebral cortex. Using 
a slightly different paradigm of lithium administration, Young et al. (2008) showed that the injection of lithium at the same time of ethanol exposure was sufficient to protect CNS neurons against ethanolinduced neuronal death. Interestingly, lithium administration $15 \mathrm{~min}$ following ethanol exposure also provided effective protection against ethanol-induced neuroapoptosis (Zhong et al., 2006; Chakraborty et al., 2008). Using a Drosophila model, French and Heberlein (2009) showed that ethanol vapors caused apoptotic degeneration of olfactory receptor neurons (ORNs) in adult Drosophila, and lithium prevented ethanol-induced death of the ORNs. Lithium was also effective in preventing ethanol-induced neuroapoptosis in vitro and inhibited ethanol-induced activation of caspase- 3 and caspase- 9 in cultured cerebellar granule neurons (Zhong et al., 2006).

In addition to ameliorating ethanol-induced neurodegeneration, lithium is also shown to reverse ethanol-induced inhibition of neuronal differentiation. We demonstrated that ethanol inhibited neurite outgrowth and the expression of neurofilaments in N2a neuroblastoma cells; lithium treatment alleviated ethanol's inhibition (Chen et al., 2009). Using an in vitro model of neural stem cell (NSC) differentiation, Ishii et al. (2008) found that ethanol inhibited neuronal differentiation of NSCs isolated from rat embryos. Lithium increased NSC differentiation and reduced ethanol-induced suppression of neuronal differentiation. Ethanol activated neuron-restrictive silencer factor (NRSF), also known as repressor element-1 silencing transcription factor (Ishii et al., 2008). NRSF is a negative transcription factor that acts at the terminal stage of the neuronal differentiation pathway to block the transcription of neuronal differentiation genes, thus having an important role in determining the fate of NSCs. Lithium decreased the DNA binding activity and protein level of NRSF enhanced by ethanol (Ishii et al., 2008). Thus, both in vivo and in vitro evidence supports that lithium is able to ameliorate ethanol's neurotoxicity.

\section{INVOLVEMENT OF GSK3 $\beta$ IN LITHIUM'S NEUROPROTECTION}

An important function of lithium is to inhibit glycogen synthase kinase 3 (GSK3). GSK3 was originally found in mammals and homologs have been found in all eukaryotes (Grimes and Jope, 2001; Doble and Woodgett, 2003). GSK3 was named for its ability to phosphorylate, and thereby inactivate, glycogen synthase, a key regulatory molecule in the synthesis of glycogen. It is now known that GSK3 plays an important role in the development of the CNS and regulates diverse early events, such as neurogenesis, neuronal migration, cell adhesion, synapse formation, neuronal survival and cell polarity/ neurite outgrowth in an immature brain (Luo, 2009). There are two highly homologous forms of GSK3 in mammals encoded by distinct genes, GSK3 $\alpha(51 \mathrm{kDa})$ and GSK3$\beta(47 \mathrm{kDa})$. Unlike most protein kinases, GSK3 is constitutively active in resting cells and undergoes a rapid and transient inhibition in response to a number of external signals (Grimes and Jope, 2001; Doble and Woodgett, 2003). GSK3 $\beta$ activity is regulated by site-specific phosphorylation. Full activity of GSK3 $\beta$ generally requires phosphorylation at tyrosine 216 (Tyr216), and conversely, phosphorylation at serine 9 (Ser9) inhibits GSK3 $\beta$ activity. Lithium inhibits the activity of both GSK $3 \alpha$ and GSK3 $\beta$ and promotes inhibitory phosphorylation at Ser 21 and Ser9 of GSK3 $\alpha$ and GSK3 $\beta$, respectively (Jope, 2003).

Available evidence indicates that ethanol can activate GSK3 $\beta$ in neurons. We demonstrated that ethanol induced a dephosphorylation of GSK3 $\beta$ at Ser9 [p-GSK3 $\beta$ (Ser9)] in the cerebral cortex of
PD7 mice, but had little effect on the phosphorylation at Tyr216 and the expression of total GSK3 $\beta$ (Liu et al., 2009). Saito et al. (2010) showed that ethanol increased the accumulation of caspase-cleaved tau, a substrate of GSK3 $\beta$, in PD7 mice and lithium blocked ethanolmediated tau expression. The notion that lithium's neuroprotection is mediated by its inhibition of GSK3 $\beta$ is further supported by the evidence that lithium blocks ethanol-induced down-regulation of p-GSK3ß(Ser9) in PD7 mice (Chakraborty et al., 2008). Shaggy is the Drosophila homolog of GSK3 $\beta$. Like lithium, a dominant negative Shaggy is able to block ethanol-induced apoptosis of olfactory neurons in adult Drosophila (French and Heberlein, 2009). Ethanolmediated activation of GSK3 $\beta$ is also observed in vitro. We demonstrated that ethanol caused a strong dephosphorylation of GSK3 $\beta$ at Ser9 in N2a cells without affecting p-GSK3 $\beta$ (Tyr216) (Chen et al., 2009). Ethanol-induced activation of GSK3 $\beta$ was evident by an increase in the phosphorylation of tau, a substrate of GSK3 $\beta$. Similar to our observations, de la Monte and Wands (2002) demonstrated that prenatal ethanol exposure enhanced GSK3 $\beta$ activity in cultured cerebellar neurons. They also showed that ethanol activated GSK3 $\beta$ in cultured human CNS-derived primitive neuroectodermal tumor 2 (PNET2) cells (Carter et al., 2008). However, it appears in the adult brain and some other cell types, ethanol may inhibit GSK3 $\beta$. Neznanova et al. (2009) demonstrated that acute ethanol exposure, at a dose commonly regarded as reinforcing, strongly phosphorylated GSK3 $\beta$ at Ser9 in the medial prefrontal cortex, with corresponding increased phosphorylation of AKT in ethanol-preferring AA rats. They suggested the GSK $3 \beta$ pathway may be involved in high ethanol preference. In cardiac cells, ethanol increases p-GSK3 $\beta$ (Ser9) and inactivates GSK3 $\beta$ (Zhou et al., 2009).

To further establish the role of GSK3 $\beta$ in ethanol neurotoxicity, we over-expressed wild type (WT), S9A mutant or kinase deficient (KD) GSK3 $\beta$ in SK-N-MC neuroblastoma cells, a cell line relatively insensitive to ethanol exposure. The KD and S9A GSK3 $\beta$ specifically inhibited and activated GSK3 $\beta$, respectively. Over-expression of WT or S9A mutant GSK3 $\beta$ in SK-N-MC cells did not induce cell death, but greatly promoted ethanol-induced cell death of SK-N-MC cells; however, over-expression of KD GSK $3 \beta$ conferred resistance to ethanol neurotoxicity (Liu et al., 2009). The involvement of GSK3 $\beta$ in ethanol neurotoxicity is further supported by studies using specific GSK3 $\beta$ inhibitors. We showed that both lithium and TDZD-8 protected neuroblastoma cells over-expressing WT and S9A GSK3 $\beta$ from ethanol-induced cell death (Liu et al., 2009). TDZD-8 is a highly selective, non-ATP competitive inhibitor of GSK $3 \beta$; it binds to the active site of GSK3 $\beta$ (Martinez et al., 2002). Takadera and Ohyashiki (2004) showed that two GSK3 inhibitors (SB216763 and alsteropaullone) completely eliminated apoptosis of primary rat cortical neurons in culture caused by ethanol exposure. The evidence supports that enhanced GSK3 $\beta$ activity indeed contributes to ethanol-induced neuronal death and lithium protection may be mediated by its inhibition of GSK3 $\beta$. However, Zhong et al. (2006) showed that lithium protected cerebellar granule neurons from ethanol-induced apoptosis, but a selective GSK3 $\beta$ inhibitor (SB415286) did not. SB415286 is a GSK3 inhibitor structurally distinct from SB216763 and inhibits both GSK3 $\alpha$ and GSK3 $\beta$ in an ATP competitive manner (Coghlan et al., 2000). They therefore suggested that GSK3 was not involved in ethanol-induced neurodegeneration. 
In addition to mediating neuronal death, GSK3 $\beta$ also plays a role in ethanol-induced inhibition of neuronal migration and differentiation. Aspartyl-asparaginyl-beta-hydroxylase (AAH) is a substrate of GSK3 $\beta$ and regulates cell motility (Carter et al., 2008). Ethanol induces GSK3 $\beta$-dependent AAH phosphorylation, resulting in $\mathrm{AAH}$ degradation which impairs migration of neuronal cells (Carter et al., 2008). Lithium mitigates ethanol-induced AAH protein degradation and impaired motility. We showed that ethanol inhibited neurite outgrowth by activating GSK3 $\beta$ through the dephosphorylation of GSK3 $\beta$ at Ser9 (Chen et al., 2009). Inhibition of GSK3 $\beta$ activity by specific inhibitors or down-regulation of GSK3 $\beta$ by siRNA reverses ethanol-induced inhibition of neurite outgrowth.

The mechanisms underlying GSK3 $\beta$-mediated neurotoxicity are unclear. There are more than 40 substrates of GSK3 $\beta$ and many of them play an important role in neuronal survival and differentiation (Luo, 2009). Direct modifications of these downstream proteins as a result of GSK3 $\beta$ activation may induce ethanol neurotoxicity. For example, we demonstrated that ethanol activated pro-apoptotic Bax in a GSK3 $\beta$-dependent manner in the developing mouse brain and in cultured neuronal cells (Liu et al., 2009). Saito et al. (2010) showed that ethanol caused an accumulation of caspase-cleaved tau which is a substrate of GSK3 $\beta$ and has been implicated in neurodegeneration in PD7 mice. AAH is also a substrate of GSK3 $\beta$ and regulates cell motility. Carter et al. (2008) found that ethanol induced a GSK3 $\beta$-dependent AAH degradation and impaired the motility of neuronal cells.

\section{OTHER MECHANISMS OF LITHIUM-MEDIATED NEUROPROTECTION}

In addition to targeting GSK3, lithium also affects the activity of other protein kinases/phosphatases, such as PKA and PP2A (Jope, 2003; Sasaki et al., 2006; Liang et al., 2008). Lithium can directly or indirectly modulate proteins involved in neuronal survival/differentiation independent of GSK3. For example, lithium is shown to promote the action of anti-apoptotic proteins, such as HSP, BDNF, IGF, VEGF, PKC, ERK, CREB, cAMP, Bcl-2, GRP78 and PI3K/AKT. It inhibits pro-apoptotic signaling such as the expression/activation of p53, Bax and tau (Bielecka and Obuchowicz, 2008; Marmol, 2008; Machado-Vieira et al., 2009; Wada, 2009). ERK is believed to be a pro-survival factor in the developing brain, and Young et al. (2008) showed that ethanol suppressed the phosphorylation of ERK (p-ERK) in the brain of PD7 mice and lithium counteracted this effect. In their study, ethanol also suppressed p-AKT, but lithium did not counteract the suppression. They therefore suggested that lithium's protection against ethanol-induced neuronal death in the developing brain was mediated by the promotion of ERK activity but not that of AKT (Young et al., 2008). In contrast, Chakraborty et al. (2008) demonstrated that lithium blocked ethanol-induced downregulation of $\mathrm{p}-\mathrm{AKT}$ and $\mathrm{p}-\mathrm{AMPK}$ in the forebrain of $\mathrm{PD} 7$ mice. These results suggest that lithium's neuroprotection may be mediated by its promotion of anti-apoptotic signals or proteins and inhibition of pro-apoptotic proteins, which may not depend on GSK3.

\section{REFERENCES}

Archibald, S. L., Fennema-Notestine, C., Gamst, A., Riley, E. P., Mattson, S. N., and Jernigan, T. L. (2001). Brain dysmorphology in individuals with severe prenatal alcohol exposure. Dev. Med. Child Neurol. 43, 148-154.

Bielecka, A. M., and Obuchowicz, E. (2008). Antiapoptotic action of lithium and valproate. Pharmacol. Rep. $60,771-782$.

Due to its diverse targets, the action of lithium may be complex and its neuroprotective property is sometimes controversial. For example, Fornai et al. (2008) demonstrated a marked neuroprotection by lithium in a mouse model of familial ALS (G93A mouse); lithium delayed disease onset and duration and augmented the life span. These effects were concomitant with the activation of autophagy. However, two recent reports indicated that chronic treatment of lithium failed to slow down disease progression in a mouse model of familial ALS (Gill et al., 2009; Pizzasegola et al., 2009). We previously showed that lithium was effective in protecting cultured neurons against 6-OHDA-induced apoptosis (Chen et al., 2004). Lithium's protection against degeneration of dopaminergic neurons, however, was not observed in an animal 6-OHDA model (data not shown).

\section{CONCLUSIONS AND FUTURE RESEARCH}

Lithium is a FDA-approved drug for use in the treatment of bipolar disorder. Recent in vivo and in vitro studies have increasingly implicated that lithium is a neuroprotective agent efficacious in preventing apoptosis-dependent neuronal death. Available evidence indicates that lithium is able to ameliorate ethanol-induced neuroapoptosis and inhibition of neuronal differentiation in vivo and in vitro, suggesting lithium may be useful for treating FASD. However, additional studies are needed to determine whether lithium's ability to suppress developmental neuroapoptosis has any lasting consequences. For example, it is important to determine whether lithium is able to reverse ethanol-induced behavioral deficits. Lithium has not been approved nor proposed for uses targeting the developing brain. The potential harmful side effects of lithium depend on the dose, duration of treatment and age of the patient at the time of treatment. It is unknown whether lithium has a negative impact on development. Lithium may have weak teratogenic effects in humans, but this has been described only after chronic exposure of the fetus during the first trimester of pregnancy (Gille and Bannigan, 2006). It has been shown that although lithium was effective at preventing excitotoxic motoneuron death by targeting GSK3 $\beta$, this neuroprotective effect was associated with conspicuous cytopathological changes (Calderó et al., 2010), suggesting that lithium was unable to restore normal neuronal cytology. So far, there are no known toxic effects associated with exposure of either immature animals or humans to a single clinically relevant dose of lithium in late gestation or early childhood. However, before lithium administration could be recommended as a potential treatment against ethanol-induced neurotoxicity, a more complete safety evaluation is needed.

\section{ACKNOWLEDGMENTS}

I would like to thank Kimberly Bower for reading this manuscript. This research was supported by grants from the National Institutes of Health (AA015407 and AA017226).

Bookstein, F. L., Streissguth,A.P., Connor,P. D., and Sampson, P.D. (2006). Damage to the human cerebellum from prenatal alcohol exposure: the anatomy of a simple biometrical explanation. Anat. Rec. B New Anat. 289, 195-209.
Calderó, J., Brunet, N., Tarabal, O., Piedrafita, L., Hereu, M., Ayala, V., and Esquerda,J.E. (2010). Lithium prevents excitotoxic cell death of motoneurons in organotypic slice cultures of spinal cord. Neuroscience 165, 1353-1369. 
Carter, J. J., Tong, M., Silbermann, E., Lahousse, S. A., Ding, F. F., Longato, L., Roper, N., Wands, J. R., and de la Monte, S.M. (2008).Ethanol impaired neuronal migration is associated with reduced aspartyl-asparaginylbeta-hydroxylase expression. Acta Neuropathol. 116, 303-315.

Chakraborty, G., Saito, M., Mao, R. F., Wang, R., Vadasz, C., and Saito, M. (2008). Lithium blocks ethanolinduced modulation of protein kinases in the developing brain. Biochem. Biophys. Res. Commun. 367, 597-602.

Chen, G., Bower, K. A., Ma, C., Fang, S., Thiele, C. J., and Luo, J. (2004). Glycogen synthase kinase 3 beta (GSK3beta) mediates 6-hydroxydopamine-induced neuronal death. FASEB J. 18, 1162-1164.

Chen, G., Bower, K. A., Xu, M., Ding, M., Shi, X., Ke, Z. J., and Luo, J. (2009). Cyanidin-3-glucoside reverses ethanol-induced inhibition of neurite outgrowth: role of glycogen synthase kinase 3 beta. Neurotox. Res. 15, 321-331.

Clarren, S. K., Alvord, E. J., Sumi, S. M., Streissguth, A. P., and Smith, D. W. (1978). Brain malformations related to prenatal exposure to ethanol. $J$. Pediatr. 92, 64-67.

Coghlan, M. P., Culbert, A. A., Cross, D. A., Corcoran, S. L., Yates, J. W., Pearce, N. J., Rausch, O. L., Murphy, G. J., Carter, P. S., Roxbee Cox, L., Mills, D., Brown, M. J., Haigh, D., Ward, R. W. Smith, D. G., Murray, K. J., Reith, A. D., and Holder, J. C. (2000). Selective small molecule inhibitors of glycogen synthase kinase-3 modulate glycogen metabolism and gene transcription. Chem. Biol. 7, 793-803.

Danis, R. P., Newton, N., and Keith, L. (1981). Pregnancy and alcohol. Curr. Probl. Obstet. Gynecol. 4, 2-48.

de la Monte, S. M., and Wands, J. R. (2002). Chronic gestational exposure to ethanol impairs insulin-stimulated survival and mitochondrial function in cerebellar neurons. Cell. Mol. Life Sci. 59, 882-893.

Doble, B. W., and Woodgett, J. R. (2003). GSK-3: tricks of the trade for a multi-tasking kinase. J. Cell Sci. 116, 1175-1186.

Fornai, F., Longone, P., Cafaro, L., Kastsiuchenka, O., Ferrucci, M., Manca, M. L., Lazzeri, G., Spalloni, A., Bellio, N., Lenzi, P., Modugno, N., Siciliano, G., Isidoro, C., Murri, L., Ruggieri, S., and Paparelli, A. (2008). Lithium delays progression of amyotrophic lateral sclerosis. Proc. Natl. Acad. Sci. U.S.A. 105, 2052-2057.

French, R. L., and Heberlein, U. (2009). Glycogen synthase kinase-3/Shaggy mediates ethanol-induced excitotoxic cell death of Drosophila olfactory neurons. Proc. Natl. Acad. Sci. U.S.A. 106, 20924-20929.

Gill, A., Kidd, J., Vieira, F., Thompson, K., and Perrin, S. (2009). No benefit from chronic lithium dosing in a sibling-matched, gender balanced, investigator-blinded trial using a standard mouse model of familial ALS. PLoS ONE 4, e6489. doi: 10.1371/ journal.pone.0006489.

Gille, J. J., and Bannigan, J. G. (2006). Teratogenic and developmental effects of lithium. Curr. Pharm. Des. 12, 1531-1541.

Grimes, C. A., and Jope, R. S. (2001). The multifaceted roles of glycogen synthase kinase 3beta in cellular signaling. Prog. Neurobiol. 65, 391-426.

Harper, C. (2009). The neuropathology of alcohol-related brain damage. Alcohol Alcohol. 44, 136-140.

Ishii, T., Hashimoto, E., Ukai, W., Tateno, M., Yoshinaga, T., Saito, S., Sohma, H., and Saito, T. (2008). Lithium-induced suppression of transcription repressor NRSF/REST: effects on the dysfunction of neuronal differentiation by ethanol. Eur. J. Pharmacol. 593, 36-43.

Jope, R. S. (2003). Lithium and GSK-3: one inhibitor, two inhibitory actions, multiple outcomes. Trends Pharmacol. Sci. 24, 441-443.

Liang, M. H., Wendland, J. R., and Chuang, D. M. (2008). Lithium inhibits $S m a d 3 / 4$ transactivation via increased CREB activity induced by enhanced PKA and AKT signaling. Mol. Cell. Neurosci. 37, 440-453.

Liu, Y., Chen, G., Ma, C., Bower, K. A., Xu, M., Fan, Z., Shi, X., Ke, Z. J., and Luo, J. (2009). Over-expression of GSK3 beta sensitizes neuronal cells to ethanol toxicity. J. Neurosci. Res. 87, 2793-2802.

Luo, J. (2009). GSK3beta in ethanol neurotoxicity. Mol. Neurobiol. 40, 108-121.

Machado-Vieira, R., Manji, H. K., and Zarate, C. A. Jr. (2009). The role of lithium in the treatment of bipolar disorder: convergent evidence for neurotrophic effects as a unifying hypothesis. Bipolar Disord. 11(Suppl. 2), 92-109.

Marmol, F. (2008). Lithium: bipolar disorder and neurodegenerative diseases, possible cellular mechanisms of the therapeutic effects of lithium. Prog. Neuropsychopharmacol. Biol. Psychiatry 32, 1761-1771.

Martinez, A., Alonso, M., Castro, A., Pérez, C., and Moreno, F. J. (2002). First nonATP competitive glycogen synthase kinase 3 beta (GSK-3beta) inhibitors: thiadiazolidinones (TDZD) as potential drugs for the treatment of Alzheimer's disease. J. Med. Chem. 45, 1292-1299.

May, P. A., and Gossage, J. P. (2001). Estimating the prevalence of fetal alcohol syndrome. Alcohol Res. Health 25, 159-167.

Nash, K., Sheard, E., Rovet, J., and Koren, G. (2008). Understanding fetal alcohol spectrum disorders (FASDs) toward identification of a behavioral phenotype. ScientificWorldJournal 8 , 873-882.

Neznanova, O., Björk, K., Rimondini, R., Hansson, A. C., Hyytiä, P., Heilig, M., and Sommer, W. H. (2009). Acute ethanol challenge inhibits glycogen synthase kinase-3beta in the rat prefrontal cortex. Int. J. Neuropsychopharmacol. 12, 275-280.

Obernier, J.A., Bouldin, T.W., and Crews, F. T. (2002). Binge ethanol exposure in adult rats causes necrotic cell death. Alcohol. Clin. Exp. Res. 26, 547-557.

Olney, J. W., Ishimaru, M. J., Bittigau, P., and Ikonomidou, C. (2000). Ethanolinduced apoptotic neurodegeneration in the developing brain. Apoptosis 5 515-521.

Pfefferbaum, A., Adalsteinsson, E., Bell, R. L., and Sullivan, E. V. (2007). Development and resolution of brain lesions caused by pyrithiamine-and dietary-induced thiamine deficiency and alcohol exposure in the alcoholpreferring rat: a longitudinal magnetic resonance imaging and spectroscopy study. Neuropsychopharmacology 32 1159-1177.

Pies, R. (2002). Combining lithium and anticonvulsants in bipolar disorder: a review. Ann. Clin. Psychiatry 14 223-232.

Pizzasegola, C., Caron, I., Daleno, C. Ronchi, A., Minoia, C., Carrì, M. T. and Bendotti, C. (2009). Treatment with lithium carbonate does not improve disease progression in two different strains of SOD1 mutant mice. Amyotroph. Lateral Scler. 10 221-228.

Riley, E. P., and McGeem, C. L. (2005) Fetal alcohol spectrum disorders: an overview with emphasis on changes in brain and behavior. Exp. Biol. Med. (Maywood) 230, 357-365.

Saito, M., Chakraborty, G., Mao, R. F., Paik, S. M., Vadasz, C., and Saito, M. (2010). Tau phosphorylation and cleavage in ethanol-induced neurodegeneration in the developing mouse brain. Neurochem. Res. 35, 651-659.

Sasaki, T., Han, F., Shioda, N., Moriguchi, S., Kasahara, J., Ishiguro, K., and Fukunaga, K. (2006). Lithium-induced activation of Akt and CaM kinase II contributes to its neuroprotective action in a rat microsphere embolism model. Brain Res. 1108, 98-106.

Sowell, E. R., Mattson, S. N., Kan, E., Thompson, P. M., Riley, E. P., and Toga, A.W. (2008). Abnormal cortical thickness and brain-behavior correlation patterns in individuals with heavy prenatal alcohol exposure. Cereb. Cortex 18, 136-144.

Stratton, K., Howe, C., and Battagila, F. (eds.) (1996). Fetal Alcohol Syndrome: Diagnosis, Epidemiology, Prevention, and Treatment. Washington DC: National Academy Press.

Swayze, V.W. 2nd, Johnson, V.P., Hanson, J. W., Piven, J., Sato, Y., Giedd, J. N., Mosnik, D., and Andreasen, N. C. (1997). Magnetic resonance imaging of brain anomalies in fetal alcohol syndrome. Pediatrics 99, 232-240.

Takadera, T., and Ohyashiki, T. (2004). Glycogen synthase kinase-3 inhibitors prevent caspase-dependent apoptosis induced by ethanol in cultured rat cortical neurons. Eur. J. Pharmacol. 499, 239-245.

Wada, A. (2009). Lithium and neuropsychiatric therapeutics: neuroplasticity via glycogen synthase kinase-3beta, beta-catenin, and neurotrophin cascades. J. Pharmacol. Sci. 110, 14-28.

Young, C., and Olney, J. W. (2006). Neuroapoptosis in the infant mouse brain triggered by a transient small increase in blood alcohol concentration. Neurobiol. Dis. 22, 548-554.

Young, C., Straiko, M. M., Johnson, S. A., Creeley, C., and Olney, J. W. (2008). Ethanol causes and lithium prevents neuroapoptosis and suppression of pERK in the infant mouse brain. Neurobiol. Dis. 31, 355-360.

Zhong, J., Yang, X., Yao, W., and Lee, W. (2006). Lithium protects ethanol-induced neuronal apoptosis. Biochem. Biophys. Res. Commun. 350, 905-910.

Zhou, K., Zhang, L., Xi, J., Tian, W., and Xu, Z. (2009). Ethanol prevents oxidantinduced mitochondrial permeability transition pore opening in cardiac cells. Alcohol Alcohol. 44, 20-24.

Conflict of Interest Statement: The author declares that the research was conducted in the absence of any commercial or financial relationships that could be construed as a potential conflict of interest.

Received: 07 April 2010; paper pending published: 25 May 2010; accepted: 01 June 2010; published online: 28 June 2010. Citation: Luo J (2010) Lithium-mediated protection against ethanol neurotoxicity. Front. Neurosci. 4:41. doi: 10.3389/ fnins.2010.00041

This article was submitted to Frontiers in Neuropharmacology, aspecialty of Frontiers in Neuroscience

Copyright (๑) 2010 Luo. This is an openaccess article subject to an exclusive license agreement between the authors and the Frontiers Research Foundation, which permits unrestricted use, distribution, and reproduction in any medium, provided the original authors and source are credited. 